\title{
A new eliminating EOG artifacts technique using combined decomposition methods with CCA and H.P.F. techniques
}

Fadia Noori Hummadi Al-Nuaimy

Biomedical Engineering Department, University of Baghdad, Iraq

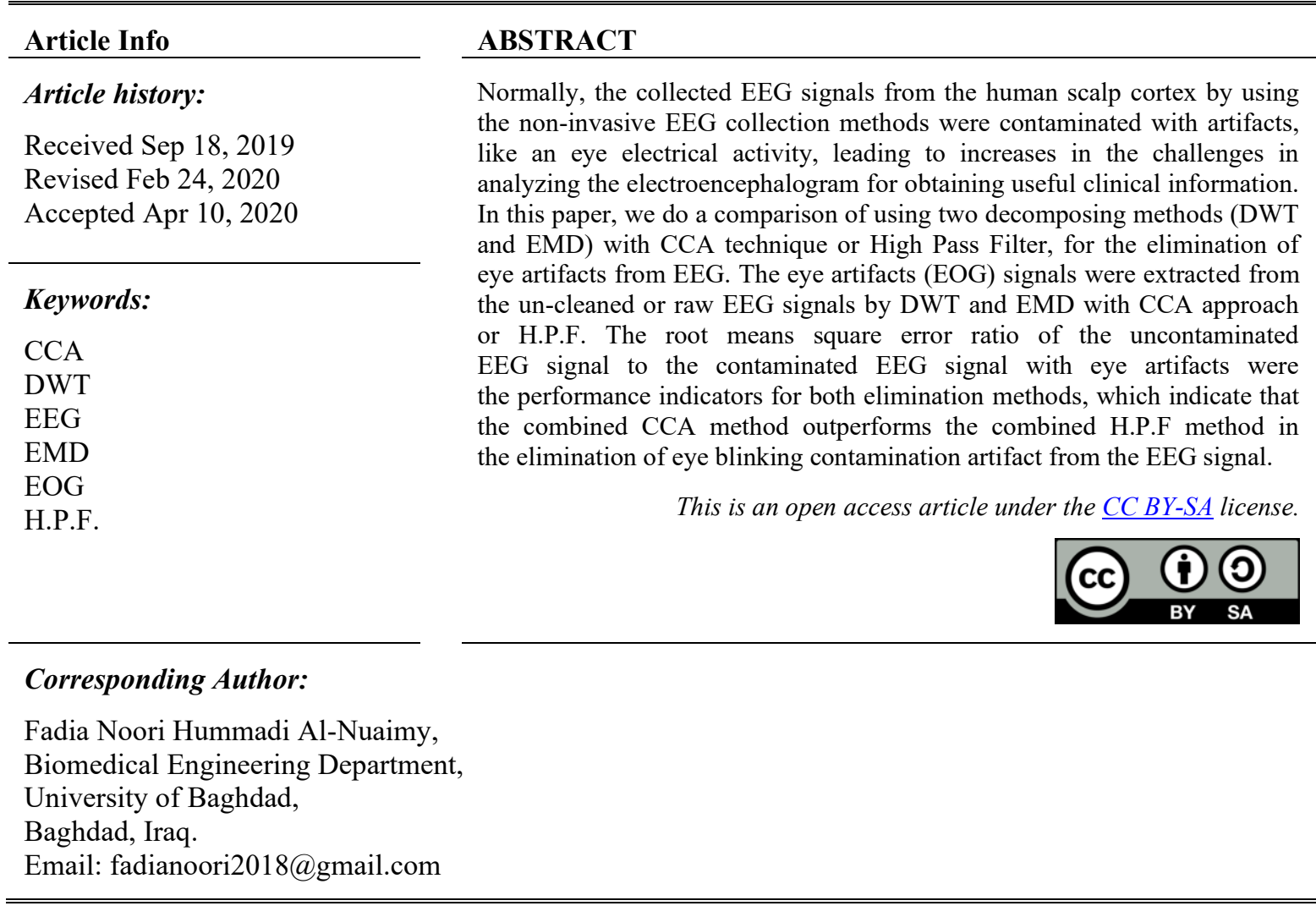

\section{INTRODUCTION}

The scalp EEG signals are usually in the range of $10-100 \mu \mathrm{V}$. These low voltage signals are easily exposed to various noise contamination [1-4]. The cleaned/pure EEG signals are widely applicable. The cleaned EEG signals are very useful in researches and to the physicians $[5,6]$. In previous studies, there are a variety of simple and advanced signal processing techniques that could be used to eliminate/remove the artifacts contaminating the EEG recorded signals. The EEG artifacts could be a simple signal that could easily be removed by simple techniques, such as low pass digital filter, bandpass digital filter, and others. These techniques are effective for cleaning EEG signals if and only if the artifacts did not lie within the influential spectrum of the useful EEG signals for reducing the power line artifacts and DC drifts.

Otherwise, the biological EEG artifacts are very difficult to remove without losing the EEG data/information during the cleaning process. This is because this type of EEG artifact shares the frequency spectrum with raw EEG signals, rendering it hard to separate from the desired spectrum. Many signal processing cleaning techniques were proposed to solve this. An advanced signal processing technique based on blind source separation (blind source separation (BSS): principal component analysis (PCA), canonical correlation analysis (CCA) [7], and ICA could be implemented to remove the eyeblink, ECG, EMG, and other biological artifacts. Other techniques such as adaptive digital filter, EMD techniques, fast fourier transform (FFT), Wavelet-based denoising are also applicable. In this study, an empirical mode decomposition (EMD) technique will be used to remove the artifact from the raw recorded EEG signals. 
In other words, an EEG signal is a record of the neuronal electrical activity that is happening as a result of the activity of many dendrites that were synoptically excited which connects a large number of pyramidal neurons in the cerebral cortex. Recordings of raw EEG signals were normally contaminants with different electrical artifact signals. These electrical artifact signals came from internal and external sources. The internal artifact sources like biological sources similar to EOG, ECG, and EMG, while external artifact sources like line power interference and or leads. The EOG generates a significant and affected artifact in the EEG signals. The vision action in the eye matched an electric dipole in which, the cornea acts as the negative part and the retina acts as the positive part. The ocular artifacts can be observed in EEG records which are the eye blinks and the eye movements which have resulted from the changes in the electrical field around the eye during movement and blinking, this is how EOG artifact signals generate.

The eye blinking can be represented by a low-frequency signal $(<4 \mathrm{~Hz})$ with a high amplitude. The eye blinking action mainly located in the front loop electrodes (FP1, FP2) and it is an asymmetrical activity with low propagation. Moreover, eye movement can also be represented by a low-frequency signal $(<4 \mathrm{~Hz})$ but with a higher propagation [8]. To eliminate or remove ocular artifacts (EOG) in the recorded EEG signals, many methods have been reported and used in the previous works, such as ICA, CCA [9]. Regression-based methods, High pass-filtering [10] and Adaptive filtering. However, some EOG artifact eliminating methods need some spatial care like ICA which, cannot be applied online [11]. Moreover, all other artifact elimination methods add complexity to the recorded system [12].

Based on that EOG artifacts generally contains from low-frequency components, therefore using a high-pass filter will be useful to remove most of the EOG's artifacts [10]. However, this EOG artifact elimination method fails when the useful EEG signal and the EOG artifact overlaps or lies in the same frequency band. In conscious of that, a simple filtering method cannot eliminate or remove the EOG signal without removing a portion of EEG [13]. Moreover, adaptive filtering is useful for the on-line removal of EOG, but, EOG's artifacts elimination methods based on adaptive filtering require extra recording channels which increases the complexity of the recorded system.

EOG signal curios are increasingly noteworthy while gathering EEG information from recording systems. These antiques can sully the nature of EEG information. What's more, on account of the volume conduction impact, both visual relics and EEG movement spread to the head surface and record by the EEG terminals or electrodes. The amplitude of EOG is generally greater than EEG signals and its frequency is similar to the frequency of EEG signals. Thus, this type of artifact (EOG) will destroy and distorted the valuable recorded EEG signals. The distorted EEG signal will do not have any useful use, it is a waste of time and effort.

Various sign preprocessing techniques to eliminate or remove eye-blink (EOG) artifact have been proposed. The common proposed technique for dispensing with antiques that are produced by eye developments using CCA and filters. However, using decomposition methods combined with CCA or filtering cleaning technique is very rare. This paper introduces two methods for the elimination of eye artifacts, which are based on the combination of DWT and empirical mode decomposition (EMD) approaches and by using CCA technique of H.P.F. for alimentation of eye artifacts.

\section{METHODS}

\subsection{Discrete wavelet transform (DWT)}

The wavelet transform (WT) is a mathematical tool able to decomposition a signal into its component scales (frequencies). Earlier examples of this success can be found in the EEG signals [14, 15].

\subsection{The empirical mode decomposition (EMD)}

The EMD is a versatile time-space examination strategy reasonable for handling arrangements that are non-stationary and non-straight. EMD performs tasks that segment an arrangement into 'modes' (IMFs; Intrinsic Mode Functions) without leaving the time area zone. It tends to be contrasted with other time-space examination strategies like fourier transforms and wavelet deterioration. Similar to these techniques, EMD did not depend on material science. In any case, the modes may give knowledge into different signs contained inside the information [16-18].

\subsection{Canonical correlation analysis (CCA)}

Canonical correlation analysis is an accepted relationship examination to distinguish and gauge the relationship among two arrangements of factors. An accepted relationship is proper in similar circumstances where different relapse would be, yet there are variations between corresponded result factors. Authoritative connection investigation decides a lot of standard variations, symmetrical direct mixes of the factors inside each set that best clarify the fluctuation both inside and between sets [19-21]. 


\section{WORK AND METHODOLOGY}

\subsection{Collecting EEG data}

In this work we collected (downloaded) signal of EEG data from a recommended well known biomedical web site (www.physionet.org), with details: sleeping EEG data with eye artifact: with (10-second duration, $\mu \mathrm{V}$ amplitude, sampling frequency 100 sample/second and FPz-Cz electrode) as its specifications.

\subsection{EEG signal decomposition}

The collected EEG data were decomposed in two steps: first using the DWT decomposition technique and secondly using EMD technique as shown below:

- Decomposition using DWT

We applied the DWT decomposing technique on the EEG that we selected to have the basic brain waves (gamma $(\delta)$, beta $(\beta)$, alpha $(\alpha)$, theta $(\theta)$ and delta $(\sigma)$ waves). The delta $(\sigma)$ wave had the lowest band frequencies according to the DWT decomposing. Thus, and since the eye artifact had lower band frequencies, we used the delta $(\sigma)$ wave in the denoising process.

- EMD decomposition

We decomposed the delta $(\sigma)$ wave using the EMD technique and getting the IMFs components. The earlier two IMFs components contain the highest frequencies since they changed rapidly (fast than others) and the eye artifacts [22].

\subsection{Elimination of eye artifacts}

Eye artifacts elimination was done via using two techniques (H.P.F. VS CCA) to compare the two techniques and find which the best are.

- Filtering using (H.P.F.):

The earlier two IMFs components of the Delta $(\sigma)$ wave was filtered by Butterworth FIR H.P.F. with the specification of ( 8 order and cutoff frequency of $2 \mathrm{~Hz}$ ) to eliminates the eye artifacts. We got the filtered two IMFs components.

- Denoising using CCA techniques

We had applied the CCA techniques for denoising on the earlier two IMFs components of the Delta $(\sigma)$ wave them from eye artifacts. We got the denoised two IMFs components.

\subsection{Reconstruction process}

We reconstructed the decomposed EEG signal into two stages as we decomposed it in two stages:

- Reconstruction of the IMFs components

We reconstructed the Delta $(\sigma)$ wave by summing the eliminated eye artifacts of IMFs components with the other IMFs components to get a clean Delta $(\sigma)$ wave from eye artifacts which are $\left(\sigma^{\prime}\right.$ and $\sigma^{\prime}$ ') for the two techniques H.P.F. VS CCA.

- Reconstruction clean from eye artifacts EEG signal

Using DWT reconstruction technique, we were reconstructed the EEG signal and we got two cleaned EEG signal from eye artifacts due to the two techniques that we had used. The paper proposed methodology block diagram is shown in Figure 1.

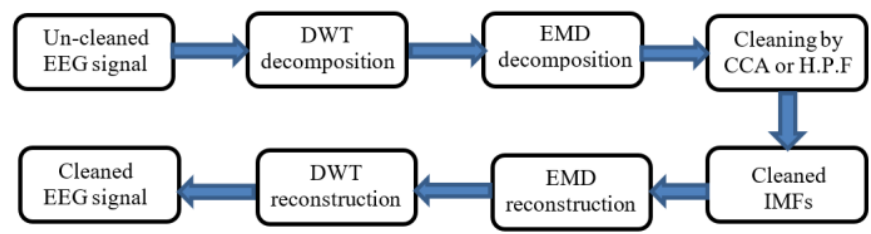

Figure 1. Proposed methodology block diagram

\subsection{Performance measurements}

For finding the performance of our two technique in the elimination of eye artifacts we measured the root mean square error (RMSE) between the raw EEG signal and the cleaned EEG signal, also we calculated two types of signal to noise ratio (SNR) for both techniques that we had used. The equation for RMSE and the two types of SNR shown:

$$
R M S E=\left(\frac{1}{N}\right) \sqrt{\sum_{n=1}^{N}\left(S^{\prime}(n)-S^{\wedge}(n)\right)^{2}}
$$




$$
\begin{aligned}
& S^{\prime} N R=10 \log ^{10}\left[\frac{\sum_{n=1}^{N} S^{2}(n)}{\sum_{n=1}^{N}\left(S \prime(n)-S^{\wedge}(n)\right)^{2}}\right] \quad(d B) \\
& S^{\prime \prime} N R=10 \log ^{10}\left[\frac{\sum_{n=1}^{N} S^{\wedge}(n)}{\sum_{n=1}^{N}\left(S \prime(n)-S^{\wedge}(n)\right)^{2}}\right] \quad(d B)
\end{aligned}
$$

where $\mathrm{S}^{\prime}(\mathrm{n})=$ raw EEG signal, $\mathrm{S}^{\wedge}(\mathrm{n})=$ resulted EEG signal, and $\mathrm{n}=1,2,3, \ldots \ldots$. So, that we can configure which cleaning technique of the two techniques that we had used in this work had better performances (i.e. given more cleanly from eye artifacts EEG signal).

\section{RESULTS}

Using the cleaning techniques shown in Figure 2 and explained in section 3, we got two cleaned EEG signal according to the cleaning techniques (CCA \& H.P.F.). Figure 2 shown the cleaned EEG signals and the un-cleaned EEF signal. The frequency spectrum of the raw EEG signal and cleaned EEG signal by CCA technique can be shown in Figure 3, also the frequency spectrum of the raw EEG signal and cleaned EEG signal by H.P.F. technique can be shown in Figure 4.

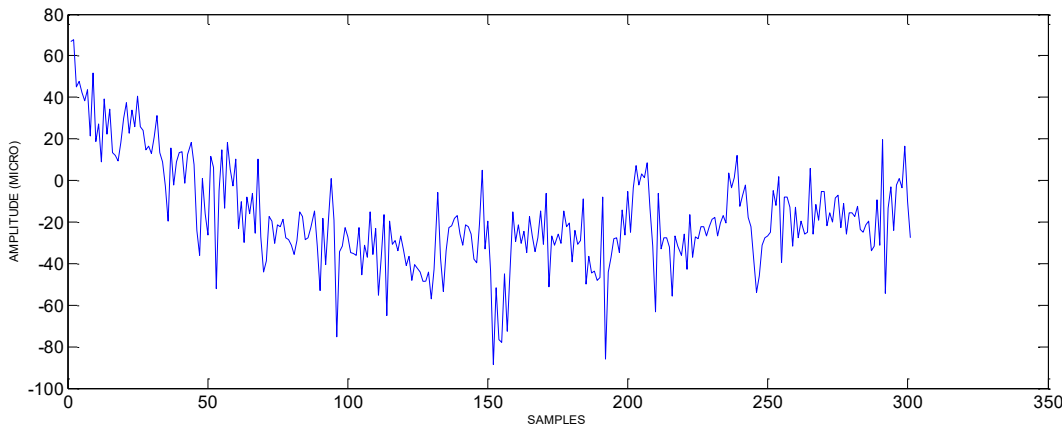

(a)

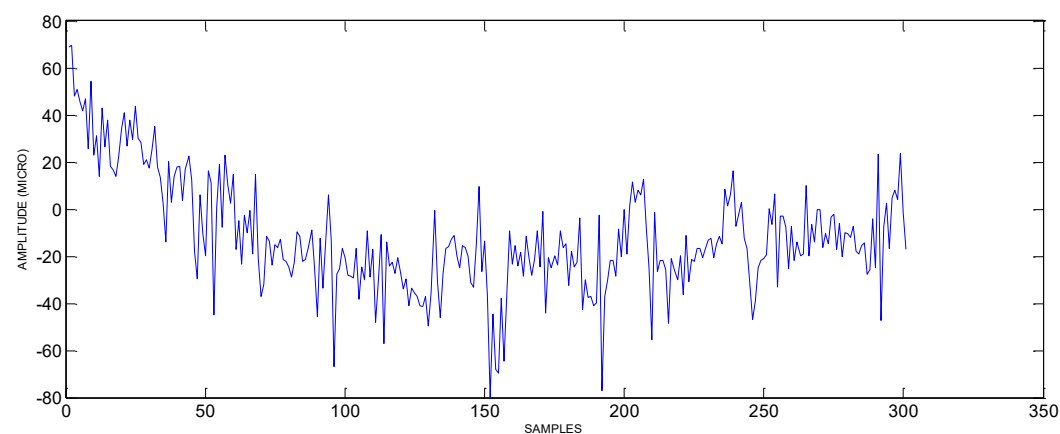

(b)

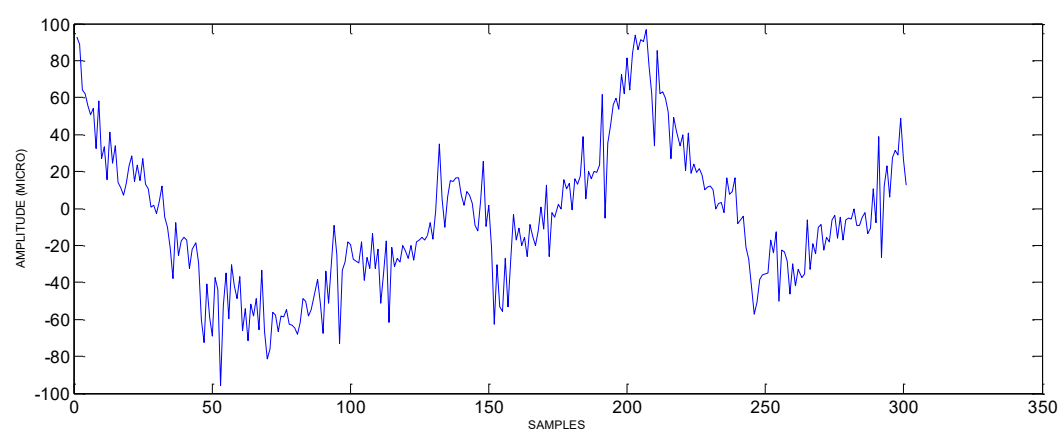

(c)

Figure 2. EEG signals; (a) EEG signal cleaned by CCA, (b) EEG signal cleaned by H.P.F., (c) Raw EEG signal 


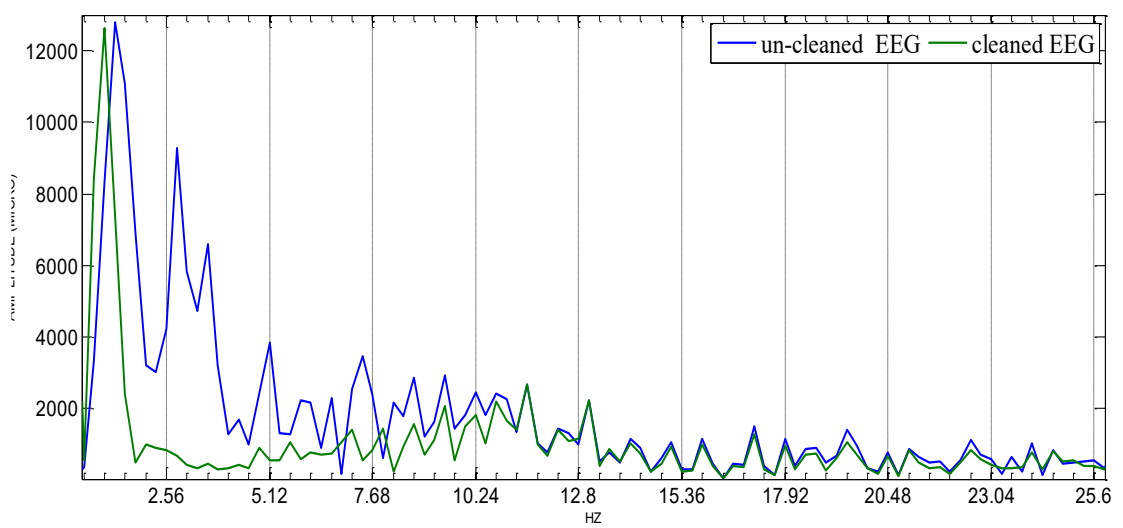

Figure 3. The spectrum of the un-cleaned and the cleaned EEG signal by CCA technique

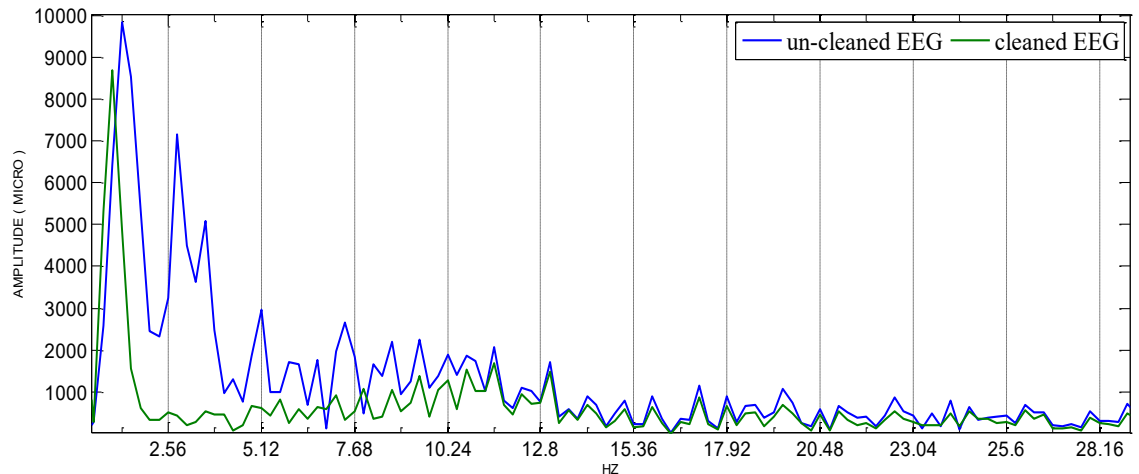

Figure 4. The spectrum of the un-cleaned and the cleaned EEG signal by H.P.F. technique

The performance of the two techniques that we were used in this work in cleaning EEG signal from eye artifacts was calculated in section 3-5 to find the (RMSE, S'NR and S"NR) for both techniques (CCA and H.P.F.) and the results were shown in Table 1. Moreover, many of EOG's artifact methods were used to eliminate the EOG artifacts that combined in the useful EEG signals, Table 1 listed some of these elimination methods results compared with the proposed methods.

Table 1. The performance measurements for EOG artifacts elimination

\begin{tabular}{cccc}
\hline Technique & RMSE & S'NR $(\mathrm{dB})$ & S"NR $(\mathrm{dB})$ \\
\hline DWT+EMD+CCA & 32.110 & 0.1889 & 1.5294 \\
DWT+EMD+H.P.F. & 29.974 & 0.7778 & 1.5051 \\
DWT+ICA & 4.2352 & 22.548 & 22.587 \\
DWT+CCA & 5.2148 & 19.854 & 20.148 \\
EMD+ICA & 11.821 & 12.358 & 11.254 \\
EMD+CCA & 12.387 & 11.647 & 11.254 \\
\hline
\end{tabular}

\section{DISCUSSION}

The commotion can introduce a critical test in examination and elucidation of EEG information, requiring effective systems for clamor aversion and expulsion. A lot of clamors can be dodged by dealing with the fitting account condition and cautious arranging of examinations and recording sessions. Moreover, various techniques and calculations can be utilized to dismiss boisterous information, evacuate commotion flag and improve the flag to-clamor proportion of the information. To successfully pick and utilize strategies for managing clamor, their points of interest, and provokes should be considered in connection to the properties of the information and the systematic inquiries being inquired. To research the execution of the proposed strategy, we contrasted this technique and another current calculation. The blend of CCA-SSA and H.P.F. (DWT-EMD) was utilized in this correlation task. To encourage the viability correlation, a similar 
EEG dataset was connected to this strategy. In the first place, by applying CCA-SSA, the crude EEG information was separated into the cerebral action and the very non-stationary EOG parts. Second, the EOG was recreated from the distinguished EOG parts by the EMD calculation.

We had used in this work two decomposition methods to looking inside the un-cleaned EEG signal deeply and can find the frequencies components with range $(<4 \mathrm{~Hz})$, then we denoised this band of frequencies by CCA technique, to eliminating eye artifacts and not harm or effect the other bands of the EEG signal. Also, we had used the H.P.F. technique for filtering the $(<4 \mathrm{~Hz})$ frequencies band of the decomposed (by the same decomposition methods above) EEG signal for eliminating eye artifacts. We did this in the order we could compare the two methodologies and find the best method for eliminating eye artifacts between them that we used. The elimination of the frequencies components with range $(<4 \mathrm{~Hz})$ in the un-cleaned EEG signal, can be seen clearly for the CCA technique in Figure 3 and H.P.F. technique in Figure 4 respectively. Thus, led to the cleaned EEG signal which was shown in Figure 2 (a) for the CCA technique and Figure 2 (b) for the H.P.F. technique compared with the un-cleaned EEG signal shown in Figure 2 (c). Furthermore, the performance measurement for cleaning EEG signals from eye artifacts, which were shown in Table 1 given us the RMSE, S'NR and S"NR for both used techniques in this work [23-25].

\section{CONCLUSIONS}

A successful methodology is proposed in this paper to address the issue of visual ancient rarity expulsion from crude EEG recording. To extricate artifactual sources from the crude EEG recording, we have utilized the stationary subspace investigation calculation, which can move relics in fewer segments than the agent dazzle source detachment strategies. At that point the artifactual parts are anticipated back to be subtracted from EEG signals, creating the spotless EEG information inevitably. The test results on both the misleadingly tainted EEG information and genuine EEG information have exhibited the viability of the proposed technique, specifically for the situations where set the number of anodes are utilized for the chronicle, just as when the antique debased flag is exceptionally non-stationary and the fundamental sources can not be thought to be free or uncorrelated.

We could have concluded from our results that using the two decomposing methods that we had used gave us a good view inside (look deeply) the un-cleaned or raw EEG signal to find the lower frequencies components of the un-cleaned EEG signal to clean it. The results have shown that the compensation of the two decomposing methods and the CCA technique had the best performance in cleaning the un-cleaned EEG signal from artifacts with $\left(\mathrm{S}^{\prime \prime} \mathrm{NR}=1.5294\right.$ and $\left.\mathrm{RMSE}=32.1101\right)$ is compared with the other way that we used here. Figures and results that we had from this work show that the proposed method (the two decomposing methods and the CCA technique) given a good cleaned from eye artifacts EEG signal.

\section{REFERENCES}

[1] V. Bono, S. Das, W. Jamal, and K. Maharatna, "Hybrid wavelet and EMD/ICA approach for artifact suppression in pervasive EEG," Journal of Neuroscience Methods, vol. 267, pp. 89-107, 2016.

[2] M. Shahbakhti, M. Bavi, and M. Eslamizadeh, "Elimination of Blink from EEG By Adaptive Filtering without Using Artifact Reference," 2013 4th International Conference on Intelligent Systems, Modelling and Simulation, pp. 190-194, 2013.

[3] M. A. Jatoi, N. Kamel, A. S. Malik, I. Faye, and T. Begum, "A survey of methods used for source localization using EEG signals," Biomedical Signal Processing and Control, vol. 11, pp. 42-52, 2014.

[4] G. J. Feist, "The psychology of science and the origins of the scientific mind: the psychology of science," Yale University Press, 2008.

[5] M. T. Al-maqtari, Z. Taha, and M. Moghavvemi, "Steady state-VEP based BCI for control gripping of a robotic hand," 2009 International Conference for Technical Postgraduates (TECHPOS), pp. 1-3, 2009.

[6] I. A. Ibrahim, F. N. AlNuaimy, and C. E. Ahmed, "Design And Implementation Of A Biomedical Signals Generator Based On Microcontroller," Journal of Engineering and Sustainable Development, vol. 17, no. 6, pp. 186-200, 2013.

[7] K. T. Sweeney, S. F. McLoone, and T. E. Ward, "The use of ensemble empirical mode decomposition with canonical correlation analysis as a novel artifact removal technique," IEEE Transactions on Biomedical Engineering, vol. 60, pp. 97-105, 2013.

[8] J. F. Gao, Y. Yang, P. Lin, P. Wang, and C. X. Zheng, "Automatic removal of eye-movement and blink artifacts from EEG signals," Brain Topography, vol. 23, pp. 105-114, 2010.

[9] G. Geetha and S. Geethalakshmi, "Scrutinizing different techniques for artifact removal from EEG signals," International Journal of Engineering Science and Technology (IJEST), vol. 3, no. 2, pp. 1167-1172, 2011.

[10] V. Krishnaveni, S. Jayaraman, S. Aravind, V. Hariharasudhan, and K. Ramadoss, "Automatic identification and Removal of ocular artifacts from EEG using Wavelet transform," Measurement Science Review, vol. 6, no. 4, pp. 45-57, 2006. 
[11] J. Gao, H. Sultan, J. Hu, and W.-W. Tung, "Denoising nonlinear time series by adaptive filtering and wavelet shrinkage: a comparison," IEEE Signal Processing Letters, vol. 17, no. 3, pp. 237-240, 2010.

[12] E. Kroupi, A. Yazdani, J.-M. Vesin, and T. Ebrahimi, "Ocular artifact removal from EEG: a comparison of subspace projection and adaptive filtering methods," 2011 19th European Signal Processing Conference, pp. 1395-1399, 2011

[13] A. Romanov, E. Sharova, O. Kuznetsova, L. Oknina, P. Volynskii, and G. Shchekutiev, "Potential of a wavelet synchronization method for assessing the long-latency components of auditory evoked potentials in healthy humans," Neuroscience and Behavioral Physiology, vol. 42, pp. 588-593, 2012.

[14] G. Kaiser, "A friendly guide to wavelets," Springer Science \& Business Media, 2010.

[15] C. Hemanth, A. S. RAM, N. Krishna, and P. Brahmanandam, "Non Linear and Non-Stationary Data Analysis using Hilbert-Huang Transform," Journal of Theoretical and Applied Information Technology, vol. 29, no. 2, pp. 74-84, 2011.

[16] M. Naji, M. Firoozabadi, and S. Kahrizi, "The Application of Empirical Mode Decomposition in Elimination of ECG contamination from EMG signals," 2011 18th Iranian Conference of Biomedical Engineering (ICBME), pp. 77-80, 2011.

[17] M. Manjula, S. Mishra, and A. Sarma, "Empirical mode decomposition with Hilbert transform for classification of voltage sag causes using probabilistic neural network," International Journal of Electrical Power \& Energy Systems, vol. 44, no. 1, pp. 597-603, 2013.

[18] C. Kufs, "Stats with cats: The domesticated guide to statistics, models, graphs, and other breeds of data analysis," Wheatmark Inc., 2011.

[19] F. Roemer, G. Del Galdo, and M. Haardt, "Tensor-based algorithms for learning multidimensional separable dictionaries," 2014 IEEE International Conference on Acoustics, Speech and Signal Processing (ICASSP), pp. 3963-3967, 2014.

[20] X. Chen, C. He, and H. Peng, "Removal of muscle artifacts from single-channel EEG based on ensemble empirical mode decomposition and multiset canonical correlation analysis," Journal of Applied Mathematics, vol. 2014, 2014.

[21] E. Noh and V. R. De Sa, "Canonical correlation approach to common spatial patterns," 2013 6th International IEEE/EMBS Conference on Neural Engineering (NER), pp. 669-672, 2013.

[22] I. A. Ibrahim, H.-N. Ting, and M. Moghavvemi, "The effects of audio stimuli on auditory-evoked potential in normal hearing Malay adults," International Journal of Health Sciences, vol. 12, no. 5, pp. 25-34, 2018.

[23] X. Jiang, G.-B. Bian, and Z. Tian, "Removal of artifacts from EEG signals: a review," Sensors, vol. 19, no. 5, pp. 987, 2019.

[24] P. Li, Z. Chen, and Y. Hu, "A method for automatic removal of EOG artifacts from EEG based on ICA-EMD," 2017 Chinese Automation Congress (CAC), pp. 1860-1863, 2017.

[25] V. Bono, S. Das, W. Jamal, and K. Maharatna, "Hybrid wavelet and EMD/ICA approach for artifact suppression in pervasive EEG," Journal of Neuroscience Methods, vol. 267, pp. 89-107, 2016. 